\title{
LC-MS/MS Assay for the Measurement of Cannabidiol Profiling in CBD Oil from Japanese Market and Application for Convertible Tetrahydrocannabinol in Acetic Acid Condition
}

\author{
Shiori Takashina, Miki Takahashi, Koji Morimoto, and Koichi Inoue* \\ College of Pharmaceutical Sciences, Ritsumeikan University; 1-1-1 Nojihigashi, Kusatsu, Shiga 525-8577, Japan. \\ Received October 19, 2021; accepted December 4, 2021
}

Cannabidiol (CBD), a major non-psychoactive cannabinoid, has a lot of attention due to its potential relaxing properties and led the trend in commercial CBD aroma/oral hemp seed oil from the Japanese market. In this study, a routine assay for evaluating CBD oil samples was performed using LC coupled with tandem mass spectrometry (LC-MS/MS) and was used to apply the convertible tetrahydrocannabinol (THC) in acetic acid conditions. Based on the electrospray positive ion mode, the detection of cannabidiolic acid (CBDA; $m / z 359>219)$, cannabigerolic acid (CBGA; $m / z 361>343)$, cannabigerol (CBG; $m / z$ 317 $>193)$, CBD $(m / z$ $315>193)$, THC $(m / z 315>193)$ and cannabinol $(\mathrm{CBN} ; m / z, 311>223)$ was performed by satisfying separation with high density of $\mathrm{C}_{18}$ column. Oil samples $(50 \mathrm{mg})$ were diluted with isopropanol $(5 \mathrm{~mL})$, to which stable isotope internal standards were added by dilution with methanol/water (50/50), and accuracy rates ranged from 97.8 to $102.2 \%$. This method was used to evaluate the CBD oil products (5 kinds) from the Japanese market. Our survey found obvious counterfeit (non-detectable CBD) CBD oil from Japanese market. Following that, we investigated the conversion of THC in CBD oil samples in simple conditions such as $\mathbf{1 0 \%}$ acetic acid and $70^{\circ} \mathrm{C}$ for $6 \mathrm{~h}$ and discovered that converts THC proportions are approximately 5\% ((THC content/CBD content) $\times 100$ ) and $<1.0 \%$. Thus, our developed LC-MS/MS assay could be applied to monitor the CBD concentration and convertible THC from CBD oil.

Key words cannabidiol; tetrahydrocannabinol; cannabidiol (CBD) oil; LC tandem mass spectrometry

\section{Introduction}

Recently, the fashioning trends of cannabis (Cannabis sativa L.), specifically cannabidiol (CBD), containing natural health cosmetics and supplements in Asia-Pacific (Japan, Australia and New Zealand) markets from increasing import growth. ${ }^{1)}$ On the other hand, in Japanese markets, CBDcontaining products are not widely available in general stores. However, specific/internet retailers selling natural health cosmetics also do business in various aspects of CBD products, such as hemp seed oil and related items advertised to help with anxiety, relaxation, restful sleeping, and anti-aging. CBD products have been appeared to be imported from the United States and other countries. In addition, the presence of the psychoactive/illegal tetrahydrocannabinol (THC) and impurity from these products have been assessed before being available to the market in Japan. ${ }^{2)}$ However, in terms of important conditions, ingredients, and storage period from common markets, it has remained insufficient that this quality evaluation of THC and/or impurity is recognized in circulated CBD products. As a result, we should investigate the possibility of impurity-containing products under a variety of conditions, including additive interaction, acid-catalyzed reaction, and unknown phenomenon. Thus, it is needed to develop the useful and simple assay for the evaluation of CBD products in Japanese markets.

The cyclization reaction of CBD seems to occur following an acid-catalyzed activation of a specific double bond. ${ }^{3,4)}$ The main products of this cyclization are $\Delta^{9}$-THC (trans $-\Delta^{9}$-tetrahydrocannabinol) and $\Delta^{8}$-THC (trans $-\Delta^{8}$ tetrahydrocannabinol). Recently, Marzullo et al. investigated the susceptibility and selectivity of CBD cyclization, differ- ent reactions, including the use of Lewis and protic acids in different solvents and varying the temperature and reaction time, concluded that $\mathrm{CBD}$ is a difficult substrate to exploit the chemical reactivity of natural alkenes and phenols. ${ }^{5)}$ Pratap Singh et al. reported that acidic cannabinoids decarboxylation kinetics in hemp seed oil based on various antioxidants. ${ }^{6)}$ Moreover, Kiselak et al. investigated to utilize a weaker acid, such as vinegar $(5.4 \%$ acetic acid) to isomerize CBD to $\Delta^{9}$-THC, $\Delta^{8}$-THC, and other impurity chemicals from original simulated-foods. ${ }^{7)}$ Unfortunately, it is impossible to obtain the useful, veridical, and reliable information about impurity-containing CBD oil from potential conditions such as coexistence in these conditions regarding to Japanese markets. Thus, we examined a LC-tandem mass spectrometry (LC-MS/MS) assay to assess the quality evaluation of CBD and cannabinoids profiles in hemp seed oil products from Japanese markets, as well as apply convertible THC in acetic acid conditions. The most frequently reported generic methods are applied with chromatographic separation such as LC-MS/MS for screening assay of CBD and other cannabinoids in various products. $^{8-12)}$ In this study, we used the previous LC-MS/MS assay to measure CBD, THC, and other cannabinoids in hemp seed oil products. ${ }^{13)}$

\section{Experimental}

Chemicals and Reagents $\Delta^{9}-\mathrm{THC} \quad(1.0 \mathrm{mg} / \mathrm{mL})$ was obtained from Sigma-Aldrich Co. (St. Louis, MO. U.S.A.). CBD $(1.0 \mathrm{mg} / \mathrm{mL})$, cannabinol (CBN) $(1.0 \mathrm{mg} / \mathrm{mL})$, cannabigerol (CBG) $(1.0 \mathrm{mg} / \mathrm{mL})$, cannabidiolic acid (CBDA) $(1.0 \mathrm{mg} / \mathrm{mL})$, cannabigerolic acid (CBGA) $(1.0 \mathrm{mg} / \mathrm{mL}), \mathrm{CBD}-$ $d_{3}(100 \mu \mathrm{g} / \mathrm{mL})$, and $\Delta^{9}-\mathrm{THCA} d_{3}(100 \mu \mathrm{g} / \mathrm{mL})$ were obtained 
from Cerilliant (St. Texas, TX. U.S.A.). FUJIFILM Wako Pure Chemical Corporation (Osaka, Japan) provided methanol, acetonitrile, isopropanol, acetic acid, and formic acid. The mobile phase was purified water, and sample preparation was accomplished using a PURELAB Flex 5 system (ELGA, U.K.). Methanol was used to adjust a stock solution of analytes $(50 \mu \mathrm{g} / \mathrm{mL})$, which was then stored at $-80^{\circ} \mathrm{C}$. In 2021 , CBD products were purchased from the Japanese market (via online shopping).

LC-MS/MS Instrument and Condition The LC system was a Waters Acquity H Class Plus (Waters Co., Milford, MA, U.S.A.). An RP analysis was performed via an Inertsil ODS-HL column $(3 \mu \mathrm{m}, 2.1 \times 150 \mathrm{~mm}, \mathrm{GL}$ Sciences, Inc., Tokyo, Japan) at $40^{\circ} \mathrm{C}$. For the optimal separation, the TSKgel ODS-100V $(3 \mu \mathrm{m}, 2.0 \times 150 \mathrm{~mm}$, Tosoh Co., Tokyo, Japan), InerSustain AQ-C18 $(1.9 \mu \mathrm{m}, 2.1 \times 150 \mathrm{~mm}$, GL Sciences, Inc.), TSKgel ODS-120H ( $3 \mu \mathrm{m}, 2.0 \times 150 \mathrm{~mm}$, Tosoh Co. $)$, Acquity UPLC BEH C18 $(1.7 \mu \mathrm{m}, 2.1 \times 150 \mathrm{~mm}$, Waters, MA, U.S.A.), TSKgel ODS-100Z $(3 \mu \mathrm{m}, 2.0 \times 150 \mathrm{~mm}$, Tosoh Co. $)$ and Atlantis T3 $(3 \mu \mathrm{m}, 2.1 \times 150 \mathrm{~mm}$, Waters $)$ were used. The injection volume was set at $5 \mu \mathrm{L}$. The mobile phase was delivered at a flow rate of $0.2 \mathrm{~mL} / \mathrm{min}$ and consisted of solvent $\mathrm{A}(0.1 \%$ formic acid in water) and solvent B ( $0.1 \%$ formic acid in acetonitrile). The linear gradient elution was as follows: $75 \%$ solvent $\mathrm{B}$ at $0 \mathrm{~min}, 75 \%$ solvent $\mathrm{B}$ at $9 \mathrm{~min}, 98 \%$ solvent $\mathrm{B}$ at $9 \mathrm{~min}$, $98 \%$ solvent $\mathrm{B}$ at $19 \mathrm{~min}, 75 \%$ solvent $\mathrm{B}$ at $19.1 \mathrm{~min}$, and $75 \%$ solvent B at $25 \mathrm{~min}$. A Waters Xevo TQD triple quadrupole mass spectrometer was operated with an electrospray ioniza- tion (ESI) source in the positive mode. The following ionization source conditions were used: capillary voltage of $2.0 \mathrm{kV}$; extractor voltage of $3 \mathrm{~V}$; RF lens voltage of $2.5 \mathrm{~V}$; cone voltage of $15-40 \mathrm{~V}$; collision energy of $15-30 \mathrm{eV}$; source temperature of $150^{\circ} \mathrm{C}$ and desolvation temperature of $400^{\circ} \mathrm{C}$. The cone and desolvation gas flows were 50 and $800 \mathrm{~L} / \mathrm{h}$, respectively, which were obtained using a nitrogen source $\left(\mathrm{N}_{2}\right.$ Supplier Model $24 \mathrm{~S}$, Anest Iwata Co., Yokohama, Japan).

Accuracy and Precision Olive oil (0.05g) samples were diluted by adding $5 \mathrm{~mL}$ of isopropanol. Then, $1.0 \mathrm{~mL}$ was diluted by adding water/methanol (1000 times dilution), added with $100 \mu \mathrm{L}$ of a mixed internal standard (detected level of $10 \mathrm{ng} / \mathrm{mL}$ ) and $1 \mathrm{~mL}$ of mixed standard solution (detected level of $500 \mathrm{ng} / \mathrm{mL})$. The accuracy value was defined as follows: $F /\left(F_{0}+A\right) \times 100(\%)$, where $F$ and $F_{0}$ are the concentrations of the analytes in the spiked and unspiked samples, respectively, and $A$ is the spiked concentration. Using olive oil, it is the $F_{0}=0$. For the precision, the same procedure was repeated for intra-day (three replicates for one day, $n=3$ for standard deviation, \pm S.D., \%).

Application for Convertible THC in Acetic Acid Condition The $0.1 \mathrm{~mL}$ of acetic acid was added into CBD oil $(1.0 \mathrm{~g})$ samples, and the sample was heated to $70^{\circ} \mathrm{C}$ on a heat block for $6 \mathrm{~h}$. After that, $50 \mathrm{mg}$ of samples were diluted with $5 \mathrm{~mL}$ of isopropanol. The $1.0 \mathrm{~mL}$ was diluted 1000 times with water/ methanol before being mixed with $20 \mu \mathrm{L}$ of a mixed internal standard (detected level of $100 \mathrm{ng} / \mathrm{mL}$ ) for LC-MS/MS assay.

Quantitative Procedure Fixed concentrations of the stan-

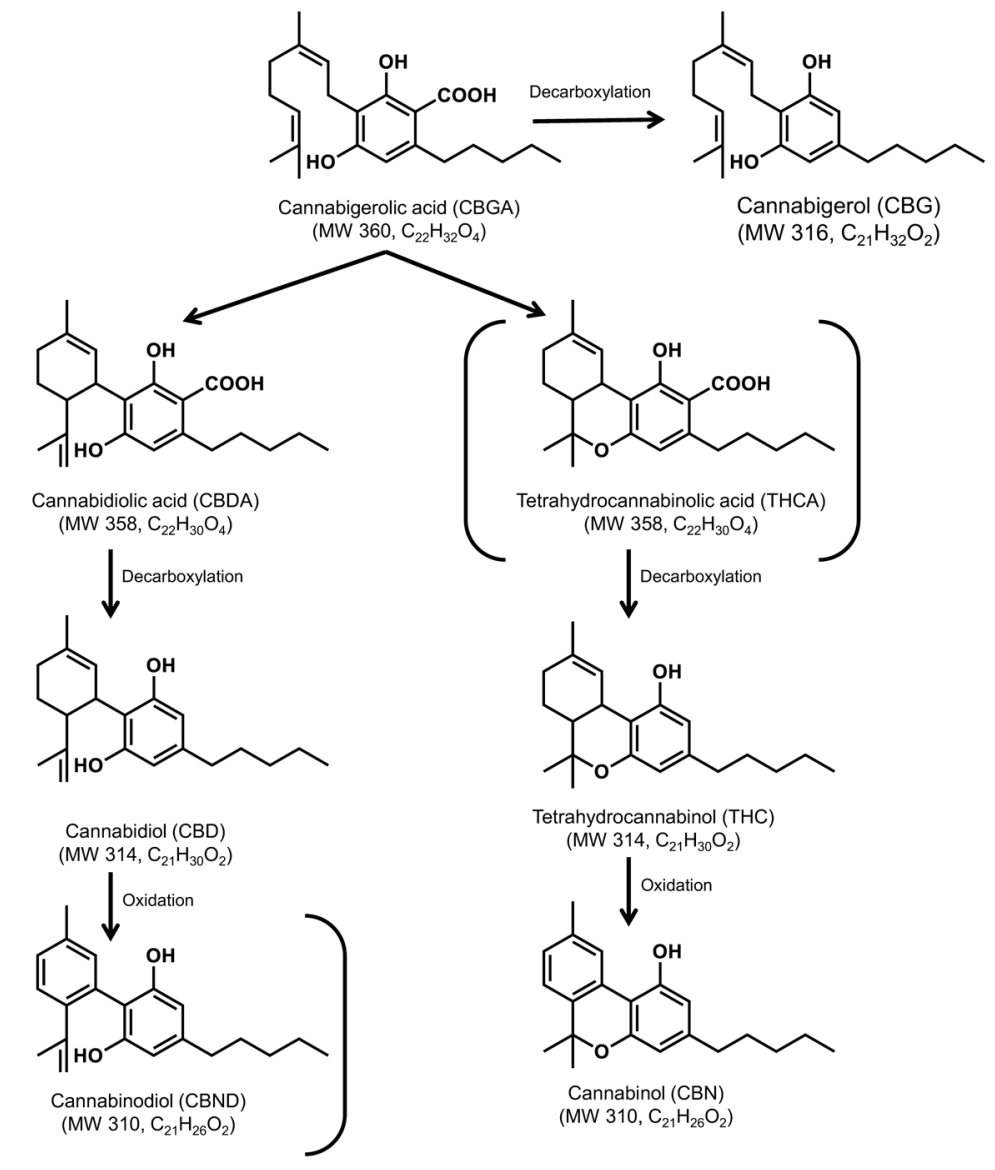

Fig. 1. Targeted Cannabidiol (CBD) Profiling in CBD Oil

Non-targeted chemicals are shown in parentheses. 


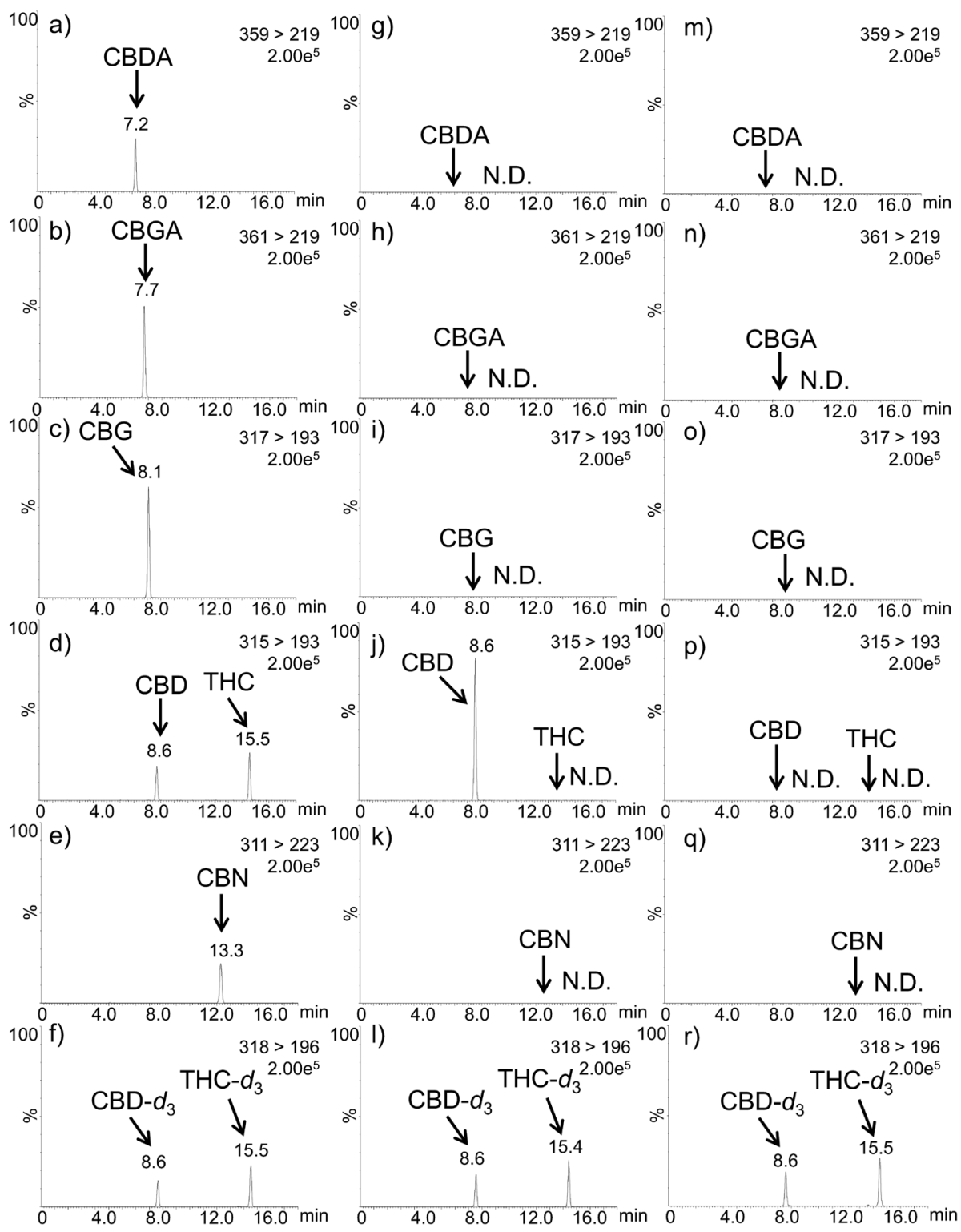

Fig. 2. Typical Selected Reaction Monitoring (SRM) Chromatograms of Targeted CBD and Other Cannabinoids

a)-f) Standard solution (125 ng/mL). g)-l) CBD oil (No. 1). m)-r) CBD oil (No. 5).

Table 1. Accuracy Test of Targeted CBD and Other Cannabinoids in Olive Oil

\begin{tabular}{lcc}
\hline \hline & \multicolumn{2}{c}{ Accuracy \pm S.D. $(\%)$} \\
\hline Analytes & Stable isotope calibration & Absolute calibration \\
\hline CBDA & $99.7 \pm 2.4$ & $100.1 \pm 3.4$ \\
CBGA & $99.6 \pm 2.3$ & $100.2 \pm 1.5$ \\
CBG & $100.3 \pm 2.7$ & $102.2 \pm 1.2$ \\
CBD & $99.9 \pm 7.1$ & $98.2 \pm 2.0$ \\
THC & $99.7 \pm 3.2$ & $101.0 \pm 0.8$ \\
CBN & $99.1 \pm 5.8$ & $97.8 \pm 3.4$ \\
\hline
\end{tabular}

For the precision, the same procedure was repeated for intra-day (three replicates for one day, $n=3$ for standard deviation, \pm S.D., $\%$ ).

dard solutions (LOQ - $1000 \mathrm{ng} / \mathrm{mL}$ ) were prepared by sequential dilutions of the stock solutions. The LOD and LOQ values were evaluated based on the signal-to-noise ratio $(S / N)$ obtained while detecting the concentration of analytes and indi-
Table 2. CBD Concentration Levels of CBD Oil from the Japanese Market

\begin{tabular}{ccc}
\hline \hline Sample No. & $\begin{array}{c}\text { Labeled value of CBD } \\
(\mathrm{mg} / \mathrm{g})\end{array}$ & $\begin{array}{c}\text { Detectable value of CBD } \\
(\mathrm{mg} / \mathrm{g})\end{array}$ \\
\hline 1 & 72.1 & 60.6 \\
2 & 55.6 & 43.9 \\
3 & 76.5 & 66.0 \\
4 & 65.6 & 57.8 \\
5 & 32.8 & N.D. $(<2.0)$ \\
\hline
\end{tabular}

cated $S / N=3$ and $S / N>10$. The calibration curves were built from eleven different concentrations to assess the linearity at each concentration level by plotting the peak-area ratio of the standard solutions: internal standard $(y)$ vs. each concentration of the adjusted standard solution ( $x$ ) (curve typesetting; linear, origin: exclude, weighting: $1 / x$, axis: none). 


\section{Results and Discussion}

LC-MS/MS Assay for CBD Profiling In this study, the targeted CBD profiling was shown in Fig. 1 and selected 6 chemicals. In this profiling, the psychoactive/illegal cannabinoids are THC and THCA in Japan. According to drug law, the THC standard solution was legally obtained from the imported
U.S.A. However, due to a procedural issue that is monitored to the SRM with the same molecule weight of CBDA $(\mathrm{m} / \mathrm{z}$ $359>219$ ), we are unable to obtain the THCA standard in the study. ${ }^{11)}$ In addition, the minor oxidized CBD (CBND) standard was not obtained from common route and monitored to the SRM with the same molecule weight of CBN $(\mathrm{m} / \mathrm{z} 311>223)$. a)

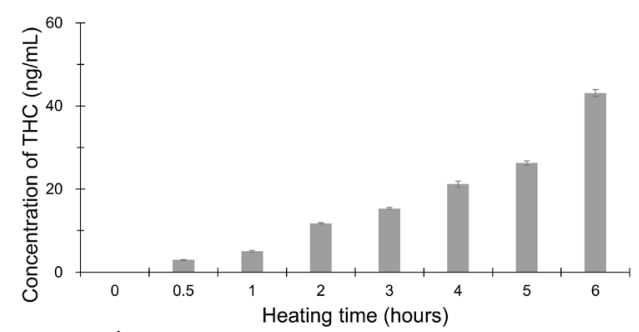

c)

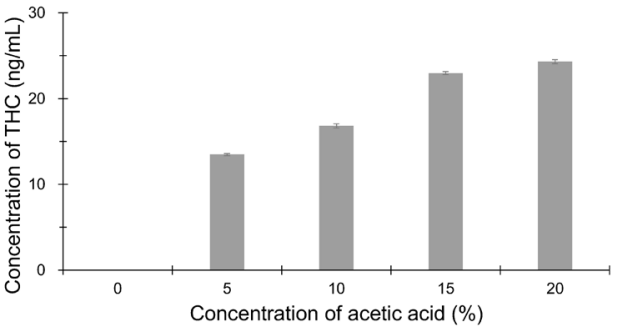

d)
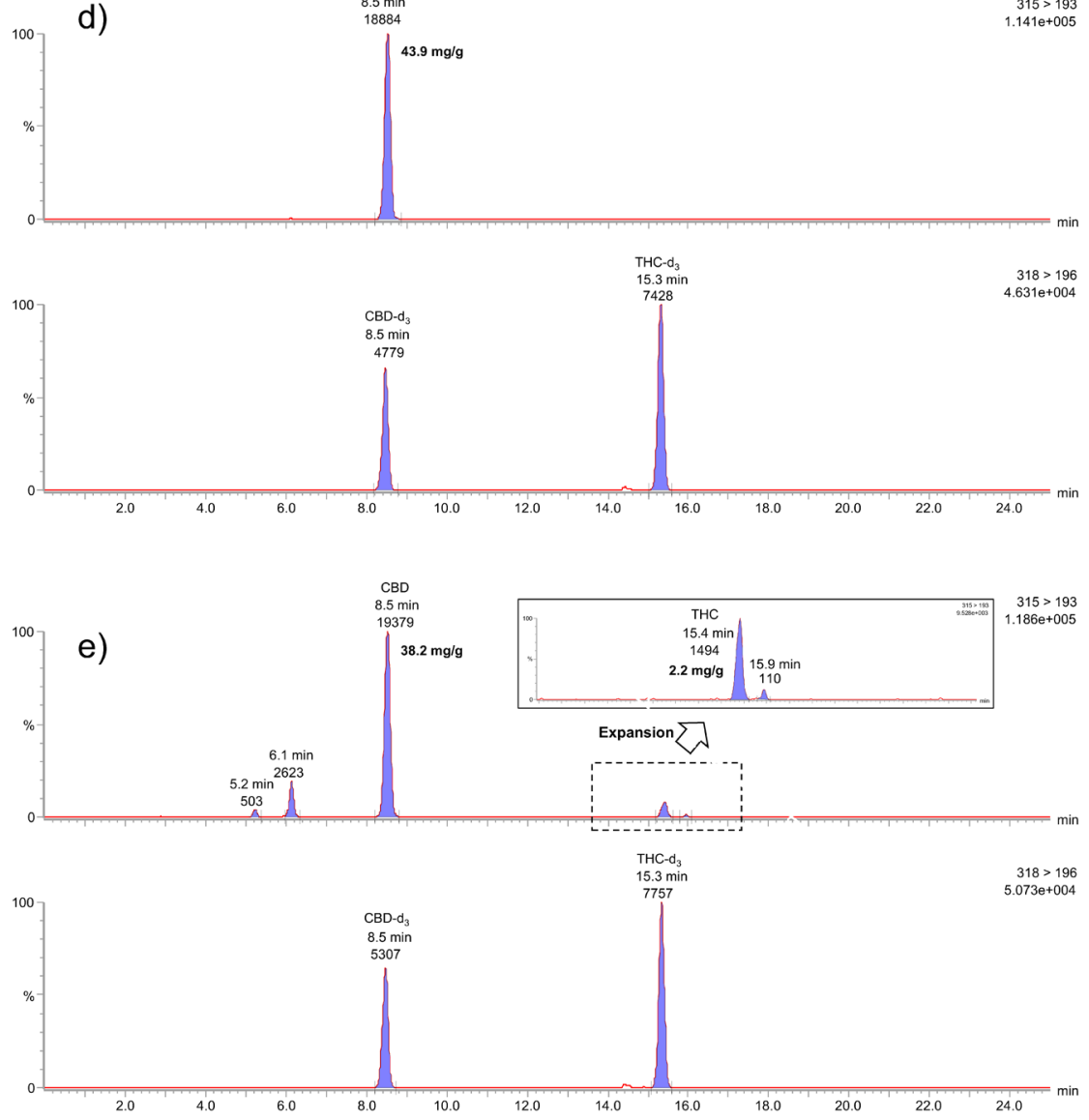

Fig. 3. Investigation of Convertible THC in Acetic Acid Condition

a) Investigation of heating time for convertible THC (three replicates for one day). b) Investigation of heating temperature for convertible THC (three replicates for one day). c) Investigation of acetic acid for convertible THC (three replicates for one day). d) SRM chromatograms (upper $m / z 315>193$ for analytes and lower $m / z 318>196$ for IS) of CBD oil (No. 2) with $10 \%$ acetic acid at room temperature for $6 \mathrm{~h}$. e) SRM chromatograms (upper $m / z 315>193$ for analytes and lower $m / z 318>196$ for IS) of CBD oil (No. 2) with $10 \%$ acetic acid at high temperature $\left(70^{\circ} \mathrm{C}\right)$ for $6 \mathrm{~h}$. 
Supplementary Table S1 shows the MS conditions such as monitoring ion, cone voltage, and collision energy. Following that, it was determined that the resolution in SRM chromatograms of CBG $(m / z 317>193)$, CBD $(m / z 315>193)$, and THC $(m / z 315>193)$ should be examined using RP columns. Specifically, these resolution values (Rs) of $\mathrm{CBG} / \mathrm{CBD}$ are performed such as TSKgel ODS-100V $(\mathrm{Rs}=0.41)$, InerSustain AQ-C18 $(\mathrm{Rs}=0.60)$, TSKgel ODS-120H $(\mathrm{Rs}=0.70)$, Acquity UPLC BEH C18 (Rs =0.86), TSKgel ODS-100Z $(\mathrm{Rs}=1.33)$, Atlantis $\mathrm{T} 3(\mathrm{Rs}=1.49)$ and Inertsil ODS-HL $(\mathrm{Rs}=1.56)$, respectively. As a result of this study, the Inertsil ODS-HL column was found to be a valid and feasible column for LC-MS/MS analysis of CBD profiling. Figures 2-a)-f) and Supplementary Table S1 shows the SRM chromatograms and quantitative performance.

Evaluation of CBD Oils For the validation test, the standard solutions were spiked in an olive oil sample $(n=3)$. The spiked olive oils were pretreated and subjected to internal standard and absolute calibrations (Table 1). These accuracy and precision values were also presented in our previous report. ${ }^{13)}$ In addition, a similar preparation using isopropanol revealed that the accuracy values of $\mathrm{CBD}$, THC, and $\mathrm{CBN}$ in olive oil ranged from 92.2 to $108.7 \%{ }^{14)}$ Secondly, the labeled CBD oil $(65.6 \mathrm{mg} / \mathrm{g})$ was analyzed that the detectable level of CBD $(57.8 \mathrm{mg} / \mathrm{g}, 88.1 \%)$ was evaluated. Using this procedure, these CBD oil samples $(n=5)$ from Japanese markets were evaluated (Table 2). Figures 2-g)-1) and $m$ ) - r) show typical SRM chromatograms of CBD oils (Nos. 1 and 5). CBDA, CBGA, CBG, THC, and CBN were not detected, and CBD was not found in one sample (No. 5) with the default label. According to the previous report, it is critical to monitor improper CBD labeling through routine assays. ${ }^{15)}$

Application for Convertible THC from CBD Oil Based on Japanese cannabis control law, it is illicit hemp that the THC is detected in CBD oil. ${ }^{16)}$ Thus, our developed LC-MS/MS assay is needed to indicate the application of detectable THC in CBD oil for the quality control. Since the 1940 s, it has been studied in a chemical reaction that converts CBD to THC that acid would catalyze a chemical change of partial CBD to other cannabinoids. ${ }^{17)}$ Subsequently, it is indicated that $\mathrm{CBD}$ would convert to $\Delta^{9}$-THC, $\Delta^{8}-\mathrm{THC}$, and $\mathrm{CBN}$ when adding acid to the $\mathrm{CBD}$ in a chemical reaction with $\mathrm{H}_{2} \mathrm{SO}_{4}$ or $\mathrm{BF}_{3}{ }^{3}{ }^{3,4)}$ Kiselak et al. reported whether a weaker acid, such as vinegar (5.4\% acetic acid) could be used to convert CBD to THC from original simulated-foods. ${ }^{7)}$ In the application for chromatographic detection of THC, we look at this conversion with simple acetic acid in CBD oil samples (No. 1-4) from Japanese market. First, the LC-MS/MS method was used to determine the optimal conditions for convertible THC from CBD standard (detectable $750 \mathrm{ng} / \mathrm{mL}$ ) based on acetic acid and temperature. In this result, a higher concentration of acetic acid and temperature offered the conversion of THC (Figs. 3-a)-c)). Thus, the useful and simple condition such as $10 \%$ acetic acid and $70^{\circ} \mathrm{C}$ for $6 \mathrm{~h}$ was performed to monitor convertible THC from CBD oil. This result showed that two patterns of convertible THC are about 5\% ((THC content/CBD content) $\times 100,4.6 \%$ for No. 2 and $6.6 \%$ for No. 3 ) and $<1.0 \%$ ( $0.5 \%$ for No. 1 and $1.3 \%$ for No. 4$)$. In addition, convertible THC from CBD oil was not detected in room temperatures for several hours. Figures 3-d), e) shows typical SRM chromatograms $(\mathrm{m} / \mathrm{z} 315>193$ for analytes and $\mathrm{m} / \mathrm{z} 318>196$ for internal standard) of CBD oil (No. 2) at room or high temperature $\left(70{ }^{\circ} \mathrm{C}\right)$ for $6 \mathrm{~h}$. Positively, standard matching estimates the peak (retention time; $15.4 \mathrm{~min}$ ) at $\Delta^{9}$-THC. In addition, the developed LC-MS/MS assay could separate $\Delta^{9}$-THC and $\Delta^{8}$-THC (retention time; $15.9 \mathrm{~min}$ ). Furthermore, based on the previous report regarding retention times and $\mathrm{MS} / \mathrm{MS}$ fragmentation compared with CBD, these peaks (retention time; $5.2 \mathrm{~min}$ and $6.1 \mathrm{~min})$ are expected of $11-5^{\prime \prime}$-dihydroxy-CBD $(\mathrm{m} / \mathrm{z} 315>193)$ and 8-OH-iso-cannabicyclohexanol $(\mathrm{m} / \mathrm{z} \quad 315>193)^{7)}$ These contents were stable in the presence of acetic acid in CBD oil for several days. The minuscule quantities of convertible THC and other cannabinoids could be separated and detected in CBD oil using developed LC-MS/MS assay. We advise that this reaction of convertible THC from CBD oil is inefficient and impurity process for abusable use. However, it is possible using LC-MS/MS assay to occur a tough decision of THC content in deteriorated CBD oil and/or various products included with food materials/additives based on the criterion value such as "not detectable THC in CBD products."

\section{Conclusion}

The LC-MS/MS assay was performed for the measurement of cannabidiol profiling in CBD oil from the Japanese market and applied for the evaluation of CBD oil and convertible THC in acetic acid conditions. For the market survey of CBD oil, our result showed that the CBD oil contained with none of CBD is found in Japanese market. Besides, there may be no or rare case of THC content in CBD oil from Japanese market. In addition, we indicated that the minuscule quantities of convertible THC and other cannabinoids from CBD oil in acetic acid and high temperature could be separated and detected using developed LC-MS/MS assay. Future analytical research should show the useful evaluation for regulatory discussion of the quality control of CBD oil and/or various products in Japanese market.

Conflict of Interest The authors declare no conflict of interest.

Supplementary Materials This article contains supplementary materials.

\section{References}

1) McGregor I. S., Cairns E. A., Abelev S., Cohen R., Henderson M., Couch D., Arnold J. C., Gauld N., Int. J. Drug Policy, 85, 102935 (2020).

2) The narcotics control department of Japan, "For those who are considering import of CBD products such as CBD oil.”: 〈https://www. ncd.mhlw.go.jp/dl_data/cbd/guidecbd_en.pdf), cited May, 2020.

3) Gaoni Y., Mechoulam R., Tetrahedron, 22, 1481-1488 (1966).

4) Mechoulam R., Hanus L., Chem. Phys. Lipids, 121, 35-43 (2002).

5) Marzullo P., Foschi F., Coppini D. A., Fanchini F., Magnani L., Rusconi S., Luzzani M., Passarella D., J. Nat. Prod., 83, 2894-2901 (2020).

6) Pratap Singh A., Fathordoobady F., Guo Y., Singh A., Kitts D. D., Sci. Rep., 10, 10567 (2020).

7) Kiselak T. D., Koerber R., Verbeck G. F., Forensic Sci. Int., 308, 110173 (2020).

8) Pacifici R., Marchei E., Salvatore F., Guandalini L., Busardò F. P., Pichini S., Clin. Chem. Lab. Med., 55, 1555-1563 (2017).

9) Brighenti V., Licata M., Pedrazzi T., Maran D., Bertelli D., Pellati F., Benvenuti S., J. Chromatogr. A, 1597, 179-186 (2019).

10) Meng Q., Buchanan B., Zuccolo J., Poulin M. M., Gabriele J., Ba- 
ranowski D. C., PLOS ONE, 13, e0196396 (2018).

11) Nemeškalová A., Hájková K., Mikulů L., Sýkora D., Kuchař M., Talanta, 219, 121250 (2020).

12) McRae G., Melanson J. E., Anal. Bioanal. Chem., 412, 7381-7393 (2020).

13) Takashina S., Igarashi Y., Takahashi M., Kondo Y., Inoue K., Anal. Sci., 36, 1427-1430 (2020).

14) Jang E., Kim H., Jang S., Lee J., Baeck S., In S., Kim E., Kim Y. U., Han E., Forensic Sci. Int., 306, 110064 (2020).
15) Gurley B. J., Murphy T. P., Gul W., Walker L. A., ElSohly M., J. Diet, 17 (Suppl.), 599-607 (2020).

16) Ministry of Health, Labour and Welfare, Japan, "About THC included product.”: 〈https://www.mhlw.go.jp/stf/seisakunitsuite/bunya/ kenkou_iryou/iyakuhin/yakubuturanyou/other/torishimari_00004. html, cited February, 2021.

17) Adams R., Pease D., Cain C., Baker B., Clark J., Wolff H., Wearn R., J. Am. Chem. Soc., 62, 2245-2246 (1940). 